92 Department of Computer Science and Christopher S. Bond Life Sciences Center, 10 University of Missouri, Missouri, USA

$11{ }^{3}$ College of Plant Sciences and Key Laboratory of Zoonosis Research, Ministry of 12 Education, Jilin University, Jilin, China

\section{Large-scale miRNA-Target Data Analysis to Discover miRNA Co-regulation Network of Abiotic Stress Tolerance in Soybeans} Haowu Chang ${ }^{1,2 \#}$, Tianyue Zhang ${ }^{1 \#}$, Hao Zhang ${ }^{1,2^{*}}$, Lingtao $\mathrm{Su}^{2}$, Qing-Ming $\mathrm{Qin}^{3}$, Guihua $\mathrm{Li}^{3}$, Xueqing $\mathrm{Li}^{1}$, Li Wang ${ }^{1}$, Tianheng Zhao ${ }^{1}$, Enshuang Zhao ${ }^{1}$, Hengyi Zhao ${ }^{1}$, Yuanning Liu ${ }^{1,2}$, Gary Stacey ${ }^{4}$, Dong $\mathrm{Xu}^{2 *}$

${ }^{1}$ College of Computer Science and Technology \& Symbol Computation and Knowledge Engineering, Ministry of Education, Jilin University, Jilin, China

${ }^{4}$ Division of Plant Sciences and Technology, Christopher S. Bond Life Sciences Center, University of Missouri, Columbia, MO 65211, USA.

*Corresponding author: Hao Zhang zhangh@jlu.edu.cn, Dong Xu xudong@missouri.edu

${ }^{\#}$ These authors contributed equally to this work

H.Z. and D.X. conceived and designed the study; L.S., Q.Q., G.L., X.L., T.H.Z. and L.W. assembled the data; H.C. and T.H.Z. performed the analyses; H.C. wrote the modeling code; Y.L., H.W. and G.S. assisted with interpretation of the results; H.C. and T.Y.Z. wrote the manuscript; H.Z., D.X. and G.S. reviewed and revised the manuscript. The authors declare no competing interests. 


\section{Abstract}

Although growing evidence shows that microRNA (miRNA) regulates plant growth and development, miRNA regulatory networks in plants are not well understood. Current experimental studies cannot characterize miRNA regulatory networks on a large scale. This information gap provides a good opportunity to employ computational methods for global analysis and to generate useful models and hypotheses. To address this opportunity, we collected miRNA-target interactions (MTIs) and used MTIs from Arabidopsis thaliana and Medicago truncatula to predict homologous MTIs in soybeans, resulting in 80,235 soybean MTIs in total. A multi-level iterative bi-clustering method was developed to identify 483 soybean miRNA-target regulatory modules (MTRMs). Furthermore, we collected soybean miRNA expression data and corresponding gene expression data in response to abiotic stresses. By clustering these data, 37 MTRMs related to abiotic stresses were identified including stress-specific MTRMs and shared MTRMs. These MTRMs have gene ontology (GO) enrichment in resistance response, iron transport, positive growth regulation, etc. Our study predicts soybean miRNA-target regulatory modules with high confidence under different stresses, constructs miRNA-GO regulatory networks for MTRMs under different stresses and provides miRNA targeting hypotheses for experimental study. The method can be applied to other biological processes and other plants to elucidate miRNA co-regulation mechanisms.

\section{Introduction}

The growth and development of crops are often restricted due to various environmental stresses, which can lead to poor harvests and yields below their genetic potential $[1,2]$. In the past decade, microRNAs (miRNAs) have been identified as important gene expression regulatory factors that play an essential role in plant growth 
and development [3]. miRNA can target multiple genes, and multiple miRNAs can also target the same gene. miRNAs are involved in the expression of stress-responsive genes and the plant's ability to adapt to environmental change [4]. Different stresses can induce differential expressions of corresponding miRNAs in plants, while some miRNAs can respond to several abiotic stresses simultaneously [5-7]. Therefore, studying the cooperative relationship among miRNAs and the interactions with their target genes is important for understanding the role of miRNAs in controlling plant growth and development.

miRNAs may respond to adverse effects on plant growth and development, such as drought, salinity, temperature and other abiotic environmental factors. It was shown that willow leaves exposed to drought or high temperature induce differential expressions of some miRNAs [8]. For example, miR169c plays a negative regulatory role under drought stress by inhibiting the expression of its target gene nuclear factor Y-A (NF-YA) [9]. miR172a [10] and miR172c [11] endow plants with a tolerance to salt stress and water deficiency. Meanwhile, miRNAs also indirectly respond to abiotic stress by regulating other biological macromolecules. For example, miR398c can negatively regulate multiple peroxisome-related genes (GmCSD1a/b, GmCSD2a/b/c and GmCCS) and affect the drought tolerance of the soybean [12]. miR399a/b participates in developing resistance to salt stress [13]. miR5036, miR862a and miR398a/b respond to phosphorus starvation and salt stress [14]. Furthermore, miR166k/o, miR390g, and miR396c/k mediate BX10 (Al-tolerant genotype) root elongation, and miR169r triggers the BD2 (Al-sensitive B genotype) oxidative stress, which in turn triggers a different type of plant aluminum tolerance between BX10 and BD2 [15]. This indicates that miRNA may regulate plant growth under abiotic stress through a complex network. However, current studies typically explore the role of a single miRNA in response to abiotic stresses. How multiple miRNAs work together as a co-regulatory mechanism has not been significantly explored.

Several studies have uncovered interesting miRNA interactions. For example, it was 
77 shown that miRNA lin-4 and let-7 act cooperatively in Drosophila [16], while miR375, $78 \mathrm{miR} 124$ and let7b coordinately regulate Mtpn expression in mammals [17]. Similarly, the 79 miRNAs, miR125a, miR125b and miR20, work together to down-regulate erbB2/erbB3 80 in breast cancer cells $[18,19]$. Furthermore, compared with individual miRNAs alone, the 81 combination of miR16, miR34a and miR106b results in a stronger immortalized human mammary epithelial cell cycle arrest [20]. It was also found that miR21, miR23a and miR27a are three cooperative repressors in a network of tumor suppressors [21]. The same combination effects also exist in plants. For example, miR160 and miR167 are involved in the adventitious root program of Arabidopsis [23]. miR156 and miR172 play a role in the transition of soybean nutrition [24], and miR172 also plays a role in

87 flowering by inhibiting AP2 [25]. Transgenic studies of miR482, miR1512 and miR1515 showed that their over-expression may lead to a substantial increase in the number of soybean nodules [26]. Another study verified networks of 365 tissue-specific miRNA-target interactions (MTIs) [27]. The synergistic effects of miRNAs provide a new systematic perspective for the entire microRNome [23], which calls for a global analysis of miRNA-target interactions.

With the growing miRNA-target data, several methods have been developed and applied to explore this field. Shalgi et al. first constructed a miRNA network from the target genes predicted by PicTar and TargetScan [28]. Xu et al. constructed a human miRNA-miRNA functional synergy network through co-regulation functional modules [29]. Meanwhile, biclustering was also applied for two different types of objects (gene and miRNA in this case) belonging to the same cluster. In the past two decades, various

100 [33], QUBIC [34] and FABIA [35] are some commonly used general algorithms. 101 Contiguous column coherent (CCC) biclustering [36-41] and LateBiccluster [39] are 102 designed for temporal data analysis. BicPAM [40, 41], BicNET [42] and MCbiclust [43] 
103

104

105

106

107

108

109

are the latest tools. Gianvito et al. applied the biclustering algorithm to predict human miRNA-mRNA modules [47]. The application of biclustering algorithms and miRNA-target regulation module (MTRM) mining is feasible and important for analyzing miRNA regulation mechanisms. Compared with traditional clustering methods, such as Bimax [103] and BiBit [69], CUBiBit [57] shortened the computing time and provided an optimized method for finding modules in larger data. However, the result obtained by CUBiBit was mostly a fully-connected bipartite graph, and the relationship between miRNA and the target gene is complex and interactive.

In this study, we analyzed the relationship between the miRNA regulatory modules in response to abiotic stresses in the soybean as a means for extending our understanding of soybean resistance mechanisms. Previously, $\mathrm{Xu}$ et al. provided a soybean miRNA-gene network, SoyFN, based on predicted miRNA targets [49]. However, this work was based only on sequence comparisons, which may result in a high false discovery rate. In contrast, in our work, we collected experimentally proven miRNA-target relationships based on degradome sequencing in the soybean, as well as the stringent homologs of miRNA-target pairs in Arabidopsis thaliana and Medicago truncatula. Based on these reliable miRNA-target data, we performed a biclustering analysis, and iteratively fused the overlapping biclusters based on the SoyNet network to obtain the soybean miRNA-target regulatory modules in response to abiotic stresses. We provide soybean MTRMs with high confidence relevant to various stresses and present the miRNA-GO regulatory networks of these modules. Capturing these miRNA-target modules with biological significance expands our understanding of the complex regulatory mechanisms of miRNA. The methods used should be easily applicable to other plant and animal systems where sufficient data exists to perform the analyses. 


\section{Results}

130 We obtained 90,064 confirmed soybean MTIs based on multiple experimental data 131 sources and 1,189 potential soybean MTIs based on homology to experimental data from 132 Arabidopsis thaliana and Medicago truncatula. A multi-level iterative bi-clustering 133 analysis resulted in 483 soybean miRNA-target regulatory modules. We analyzed the 134 enrichment results of the top five biclusters. In addition, we identified 37 abiotic 135 stress-related modules and predicted the underlying miRNA regulatory pathway 136 networks.

\section{Identification of MTIs}

We collected soybean miRNA-target data based on databases and related publications. First, we gathered all the soybean miRNA-target interactions (MTIs)

141 verified by degradome sequencing and biological experiments by mining published data, 142 including 14,958 pairs of MTIs from DPMIND [50], 86,427 pairs of MTIs from starBase 143 [51], and 38 pairs of MTIs from TarBase [52]. Additional data came from fourteen 144 publications [70-83], as shown in Supplemental Table 1 (Supplemental Table S1). A total 145 of 111,650 pairs of soybean MTIs were obtained. After removing 21,586 redundant pairs 146 of MTIs, 90,064 pairs remained.

147 To expand MTIs, we predicted the target relationship between potential miRNAs and 148 targeting genes from the MTIs of Arabidopsis thaliana and Medicago truncatula based 149 on homology. For Arabidopsis MTIs, 43 pairs were collected from TarBase, 66,212 pairs 150 from starBase, 4682 pairs from DPMIND, 106 pairs from miRTarBase, and 369 pairs 151 from 5 related publications [84-88], resulting in a total of 71,412 pairs of Arabidopsis 152 MTIs, in which 12,094 MTIs became unique after removing 59,318 pairs of redundant 
MTIs. For Medicago MTIs, there were 302 pairs from TarBase, 22,010 pairs from starBase, 781 pairs from DPMIND, and 1,349 pairs from three publications [89-91], resulting in a total of 24,442 pairs of MTIs, in which 4,394 MTIs became unique after removing 20,048 pairs of redundant MTIs. There are 33 miRNAs with identical sequences between Arabidopsis and the soybean, covering 3,415 targeting genes in Arabidopsis and 4,190 genes in the soybean. There are also 49 miRNAs with identical sequences between Medicago and the soybean, covering 1,180 targeting genes in Medicago and 5,096 genes in the soybean. We removed any redundant MTIs resulting from these. We further validated homology-based MTIs using three miRNA target prediction tools that performed well in general plants, i.e., psRNAtarget [53, 54], TAPIR [55], and Targetfinder [56]. The relationship between a specific miRNA and its target was validated by at least one of the prediction tools. In the Arabidopsis derived MTIs, 943 pairs of MTIs were validated in the psRNAtarget, 152 pairs in TAPIR, and 158 pairs in Targetfinder, confirming a total of 961 unique pairs of MTIs. In the Medicago-derived MTIs, 968 pairs of MTIs were validated in the psRNAtarget, 144 in TAPIR, and 134 in Targetfinder, confirming a total of 986 unique pairs of MTIs, as shown in Supplemental Figure S1. There is a high overlap between the two sets of MTIs (Supplemental Table S2). After removing the redundant ones, a total of 1,189 pairs were used to expand soybean MTIs.

\section{miRNA-target regulatory modules}

We integrated the 90,064 soybean MTIs with the 1,189 MTIs based on homology. We removed MTIs involving genes that do not have the glyma2 ID. A total of 11,018 MTIs were removed, and the remaining 80,235 MTIs were used for analysis in the following tasks. 
We applied CUBiBit [57] for bi-clustering analysis, with the smallest scale $2 \times 2$ or $6 \times 2$ for miRNA-target modules (i.e., at least two or six target genes and at least two miRNAs in each module), resulting in $15,380(2 \times 2)$ miRNA-target modules or 2,461 $(6 \times 2)$ miRNA-target modules. We contracted the overlapping modules using a multi-level iterative fusion method based on the soybean gene relationship network (See Methods), yielding $6,577(2 \times 2)$ and $812(6 \times 2)$ soybean miRNA-target regulatory modules after removing the modules that were completely included in the preliminary clustering module.

We next merged MTRMs according to the set threshold until the level converged stably (level represents the number of iterations). The iterative fusion of each level is shown in Figure 1. We compared the iterative results at different scales. Soybean MTRMs at the $2 \times 2$ scale showed better results at level 10, which contains 2,715 MTRMs. Soybean MTRMs at the $6 \times 2$ scale showed a better effect at level 7, which contains 483 MTRMs. Comparing the cluster score based on the GO calculation between the two scales of stable convergence (Figure 1c) shows that the cluster score quality at the $6 \times 2$

193 scale is higher than that at the $2 \times 2$ level (Supplemental Table S3). Hence, we used the 194 GO enrichment analysis result on 483 soybean MTRMs obtained at the $6 \times 2$ level 7 . as shown in Figure 1(e) from the level 7 clustering results of the $6 \times 2$ scale and plotted it with the corresponding MTRMs under level 1 before the fusion, as shown in Figure 1(e) and after Figure 1(d), which is a level-7 fusion. The module (1534) is at level 1 before the

199 fusion has 2 miRNAs and 22 targeted genes. At level 7, the module (1534) fused an 200 additional three modules 1539, 622, and 1537, and each contains miR396. From the 201 perspective of targeting, the module at level 7 has more miRNA-target interactions than the one at level 1. 


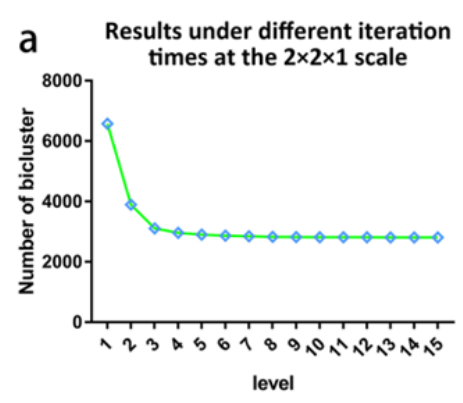

d

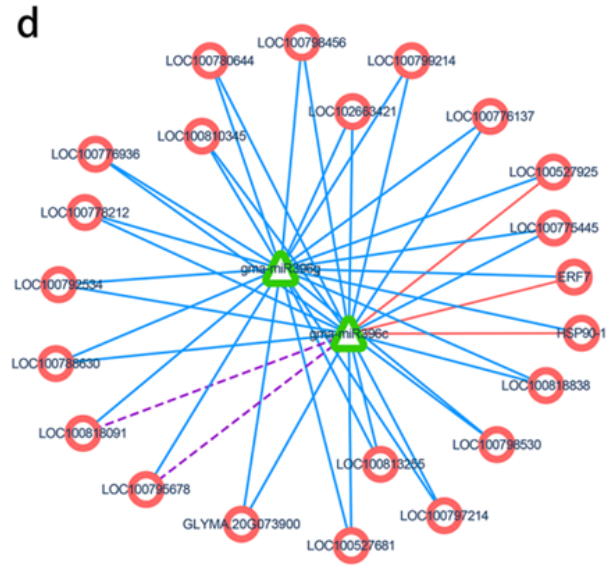

621 level 1 cluster 1534

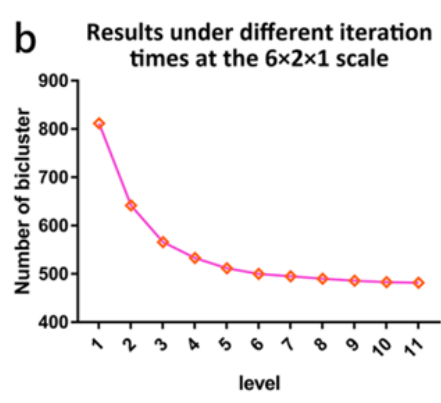

e
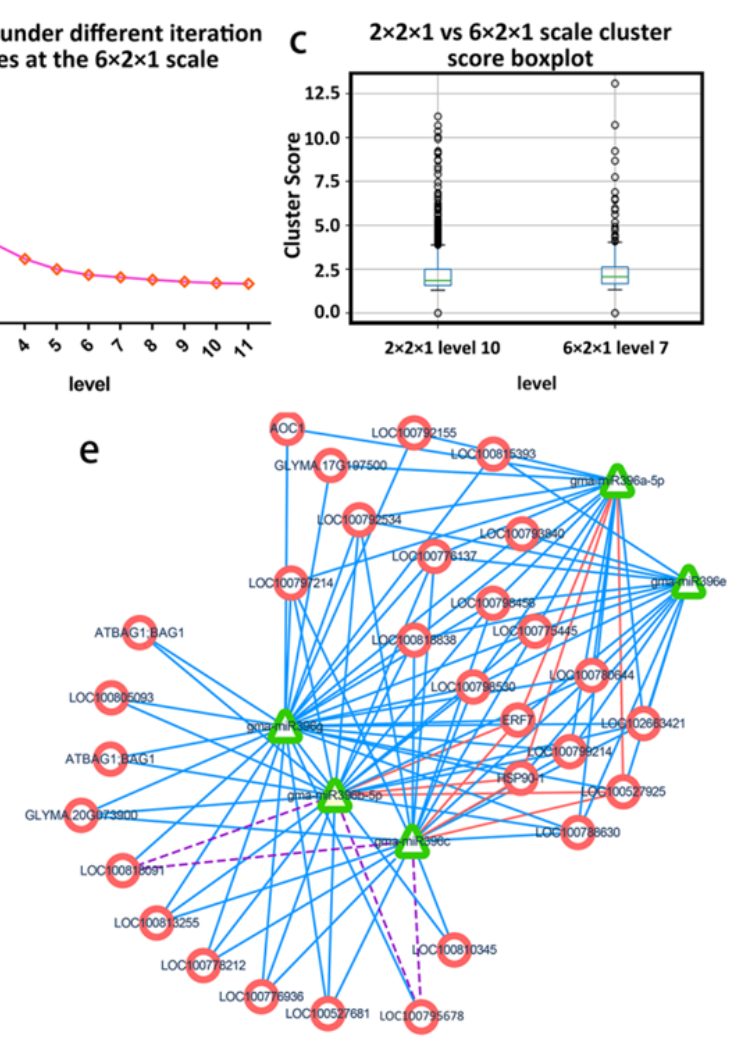

621 level 7 cluster 1534-1539-622-1537

Figure 1. Multi-level iterative biclustering results of soybean MTRMs. (a) Results under different iteration times at the $2 \times 2$ scale, (b) results under different iteration times at the $6 \times 2$ scale, and (c) the boxplot of the cluster score is calculated based on the gene ontology (GO) under the two scales when converging to a stable level, where based on the overall distribution, the results at the $6 \times 2$ scale are better; (d) shows the MTRM bicluster at level 1 before the $6 \times 2$ scale fusion, and (e) shows the corresponding MTRM bicluster at level 7 after the $6 \times 2$ scale fusion.

\section{Gene ontology (GO) analysis of MTRMs}

We screened 254 GO pathways whose GO biological processes (BP) satisfied the p-value $<0.00001$ for the GO enrichment and obtained 483 soybean MTRMs at the $6 \times 2$ scale at level 7. We analyzed the relationship among the enriched GO terms through 
217 REVIGO [122] with a parameter of 0.5. These GO pathways have a specific aggregation

218 (Figure 2a). MTRMs obtained from a global perspective have several concentrated

219 distributions of GO functions, such as cellular processes, primary metabolism, and cell

220 adhesion, as well as hormone response and negative regulation of biological processes. In

221 addition, there are metachronous positive growth regulations and chalcone biosynthesis.

222 Chalcone plays an important role in soybeans and is involved in the multi-branch

223 pathway of flavonoids and isoflavone biosynthesis [92]. The enrichment results mainly

224 involve positive regulation of development, heterochronic, chalcone biosynthesis, 225 defense response, mitochondrial mRNA modification, sulfate transport, plant-type 226 primary cell wall biogenesis, and cofactor biosynthesis, as shown in Figure 2(b).

227 In addition, we extracted the enrichment results of the top biclusters in terms of 228 cluster score among the 483 MTRMs and selected the top five GO terms of each module, 229 as shown in Figure 2(c) and Supplemental Table S4).

\section{Abiotic stress-related modules}

To explore the biological significance of soybean MTRMs, we collected related 233 soybean miRNAs, which revealed 10 types of abiotic stresses, involving 1) drought, 2) 234 salt, 3) cold, 4) nodulation, 5) $\mathrm{Pi}$, 6) oil, 7) rust, 8) soybean isoflavone, 9) pollen 235 development, and 10) phosphorus deficiency based on publications [22, 48, 105-121]. 236 The function annotations of these miRNAs are shown in Supplemental Dataset S1. In 237 most processes, soybean miRNA responses are involved in multiple abiotic responses, as 238 shown in Figure 3(a). Therefore, mining the potential cooperative regulatory modules of 239 these miRNAs is important to understand their role in modulating soybean stress 240 responses. 


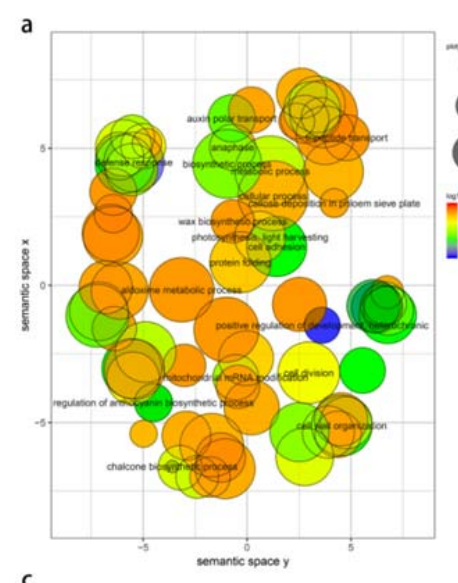

c

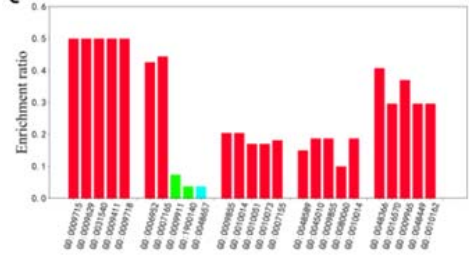

b
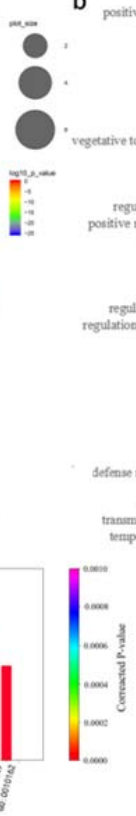

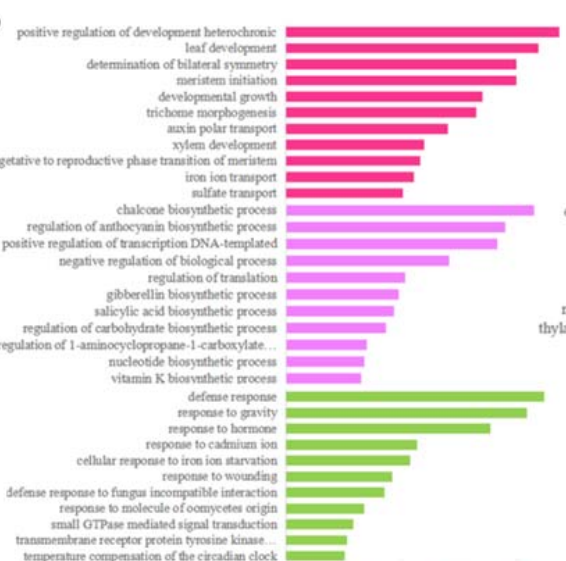

- positive regulation of development heterochronic = amaphase

- chalcone biosynthetic process

= defense response

- cell adhesion

- biosyntilitic process

- metabolic process
- cellular process

= aldoxime metabolic nomess

- cell division

= cell wall organization

= mitochondrial mRNA modification

242 Figure 2. GO analysis of soybean MTRMs. (a) Semantic relevance of GO terms wherein

243 the GO pathway has a certain concentration. (b) GO annotation enriched with 483

244 soybean MTRMs with enrichment results, which mainly involve positive regulation of

245 development, heterochronic, chalcone biosynthesis, defense responses, and mitochondrial

246 mRNA modification, and (c) GO enrichment of the top five soybean MTRMs. The listed

247 GO terms were enriched with significant $\mathrm{p}$-values $<0.00001$ 
a
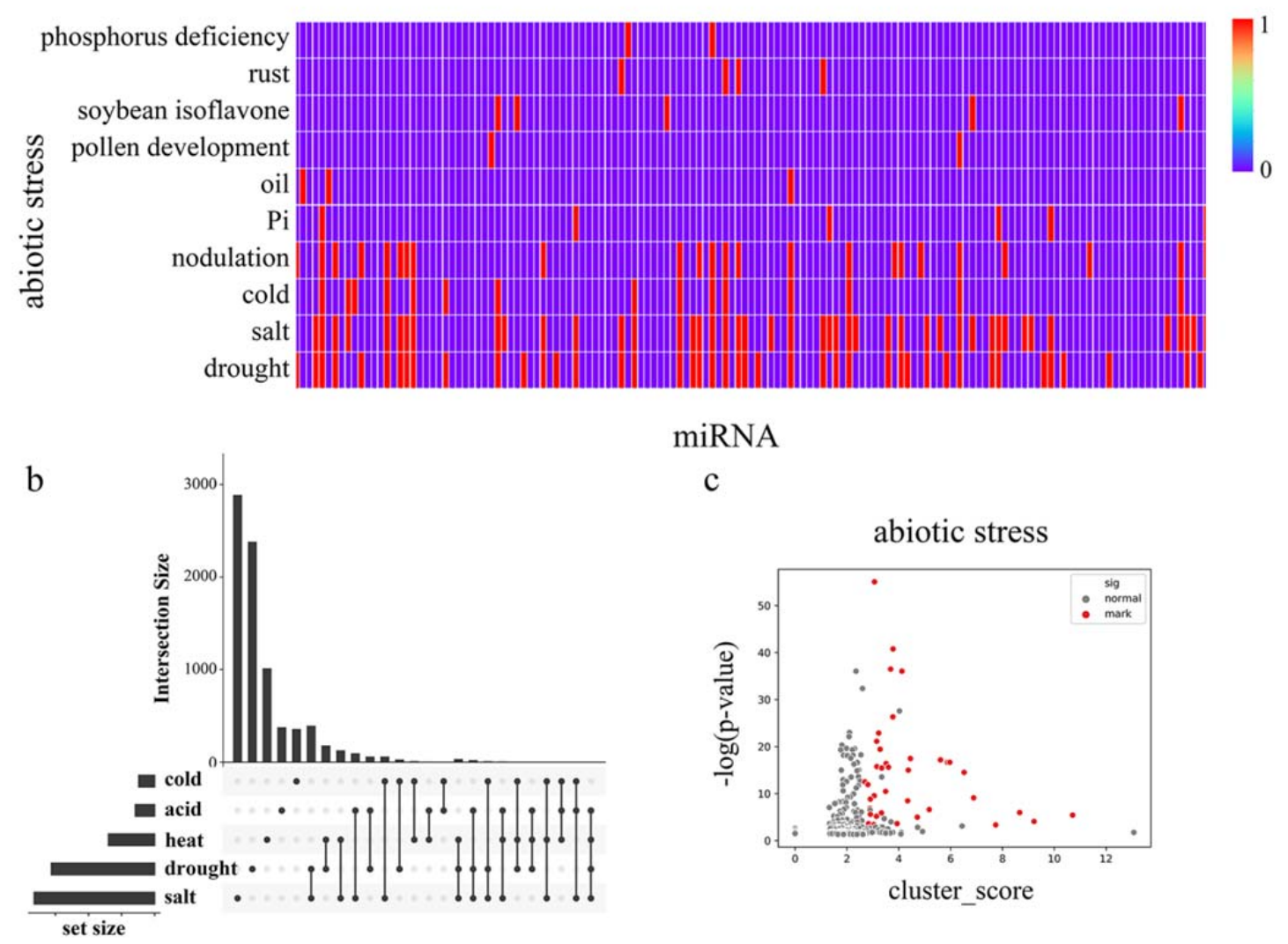

$$
\text { miRNA }
$$

C

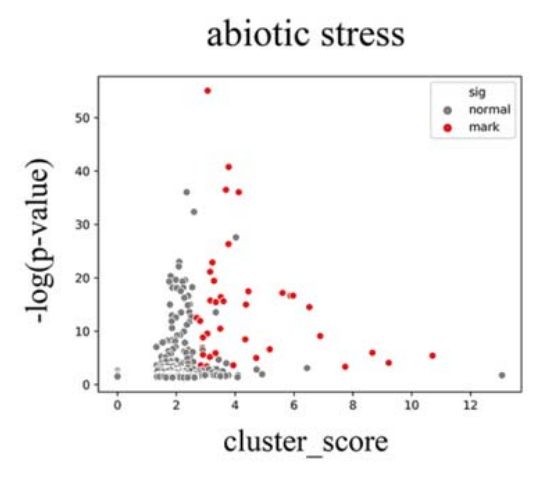

Figure 3. Collected miRNA data on soybeans involved in various abiotic stress responses based on the data statistics from the literature. (a) The distribution of stress types in miRNAs where each vertical line represents one miRNA, and red is marked as relevant, (b) UpSet diagram [123] of modular genes under various abiotic stresses within the horizontal correspondence, where dots are used to refer to the corresponding cold stress, 254 acid stress, heat stress, drought stress, and salt stress on the left. The point-to-point 255 connection is realized longitudinally to indicate the intersection between the corresponding data sets, and the upper bar graph indicates the number of genes in the

257 intersection. In (c), the differentially expressed genes in each MTRM under abiotic stress 258 are shown after screening. We used three indicators to filter the candidate clusters. 259 According to the p-value, the related miRNA purity, and the cluster score of each MTRM gene are placed under the corresponding stress. We selected the corresponding threshold 
261

262

263

264

265

266

267

268

269

270

271

272

273

274

275

276

277

278

279

280

281

282

and obtained the stress-related MTRMs with higher reliability and marked them as red dots in Figure 3(c). Supplemental Figure S2 shows MTRMs under other types of stress.

We correlated the 483 soybean MTRMs obtained by clustering with the functional annotations. We selected miRNAs that responded to drought resistance, salt resistance, heat stress, cold stress, acid stress, and performed a statistical analysis on the miRNAs in each of the 483 biclusters. We collected data on the differential expression of soybean genes in the MTRMs under drought, salt, low temperature, cold, and acid stress. Among them, the drought stress data are from GSE76636, the cold stress data are from GSE117686, the acid stress data are from GSE75575, and the salt stress expression data are from the data under $0-\mathrm{h}$ and 12-h treatments of salt stress in GSE57252 (Supplemental Dataset S2). The conditions for screening differentially expressed genes are $\log 2 \mathrm{FC}>1, \mathrm{p}<0.05$. We obtained 2,145 differentially expressed genes under soybean drought and 1,752 differentially expressed genes under salt treatment. Figure 3(b) shows the genes in the module together with an abiotic stress diagram. At the same time, we calculated the p-values and FDR. We used the Benjamin Graham formula to correct the p-value of the genes in each MTRM for the differentially expressed genes under abiotic stress scenarios through the hypergeometric distribution, as shown in Figure 3(c).

Subsequently, we screened MTRMs related to abiotic stress, drought, and salt stress according to the p-value of differentially expressed genes corresponding to the stress in the MTRMs $(\mathrm{p}<0.001$, single adversity 0.01$)$, the proportion of the corresponding miRNA family function (miR function ratio), and the cluster score (cluster score $>$ median). The screening results are shown in Supplemental Dataset S2. We obtained 37 MTRMs related to abiotic stress, including 34 MTRMs related to drought stress, 27 MTRMs related to salt stress, 3 MTRMs related to cold stress, and 21 MTRMs related to heat stress. Figure 4(a) shows the set relationship of MTRMs involved in a variety of 
stresses. The data suggest that soybean miRNAs have basic and universal functional modules in their response mechanisms to drought, high salt, high temperature, low temperature, and other abiotic stresses. There are two shared modules (31 and 493), involving 6 miRNAs and 11 miRNAs, respectively. The 6 miRNAs of module 31 belong to the miR156 family. The regulated gene-enriched GO pathway is a transcription regulation, DNA-dependent (p-value $4.24 \mathrm{e}-10$ ), and a vegetative phase change regulation with a p-value of 9.24 e- 07 . The 11 miRNAs of module 493 are mainly in the miR172 family, in addition to miR156, miR1533, miR4374, miR5782, miR3939. The regulated gene-enriched GO pathway involves an oxidation-reduction process ( $\mathrm{p}$-value = $4.66 \mathrm{e}-12)$ and a root hair elongation ( $\mathrm{p}$-value $=1.63 \mathrm{e}-08)$. Moreover, we also found stress-specific regulatory modules in our results, including 14 drought-specific MTRMs, seven salt-specific MTRMs, and two heat-specific MTRMs (Supplemental Dataset S3).

The functions of related miRNA regulatory modules under abiotic stress are mainly concentrated in positive regulation of developmental heterochrony, defense responses, cell wall organization, and other biological processes, as shown in Figure (4b). The data of the top-5 modules are shown in Supplemental Table S5.

\section{miRNA regulatory pathway network under abiotic stress}

We explored the regulatory pathway network corresponding to the miRNA of the miRNA-target regulatory module in soybean abiotic stress and analyzed the GO terms of MTRM genes under various abiotic stresses. Stringent screening conditions were used, i.e., the p-value of MTRM stress is 0.001 , and the GO BP pathway was selected with a p-value of less than $10^{-5}$. The REVIGO-based GO language correlation analysis is shown in Figure 5. GO channels with similar functions are closer in distance in the figure. The

312 of soybeans in abiotic stresses identified in this study mainly focus on resistance response, 

iron transport, positive growth regulation, and cell wall organization. Under abiotic stress, 314 the cooperating miRNA regulatory modules of the soybean mainly regulate these pathways to respond to the stress environment. The correlation analysis between drought stress and salt stress with specificity is shown in Figure 5 (b) and (c).

\section{a}

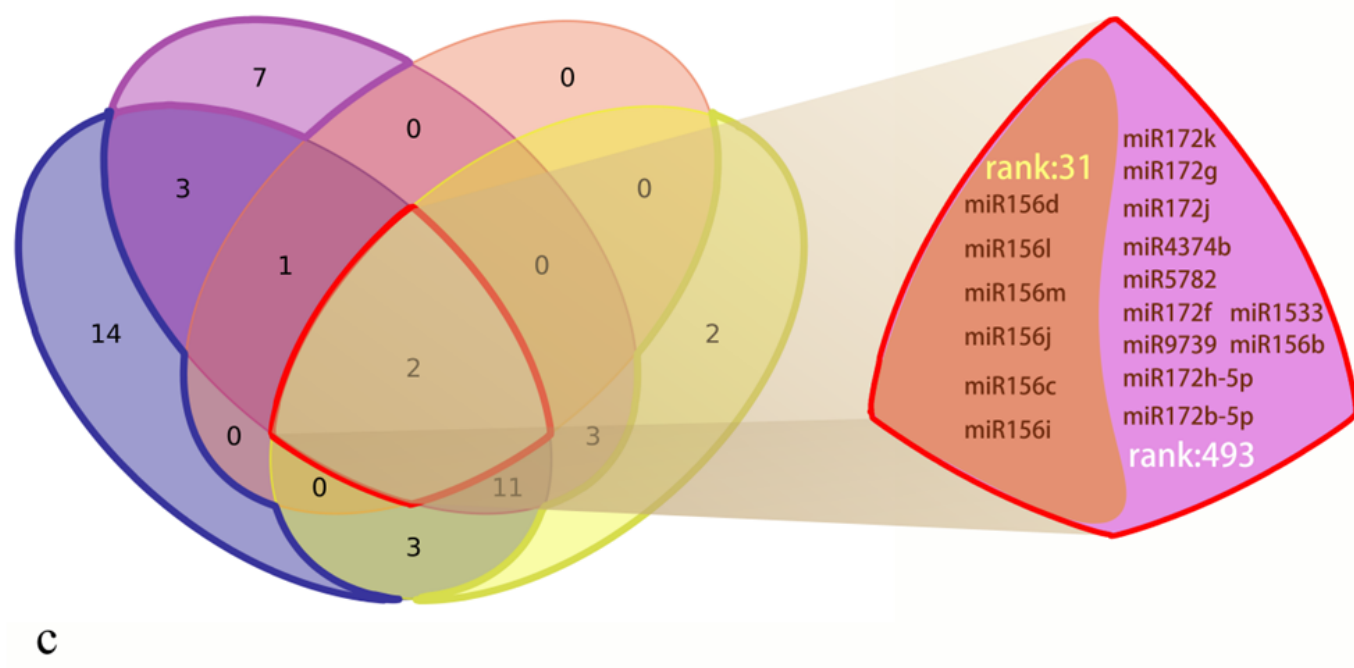

C

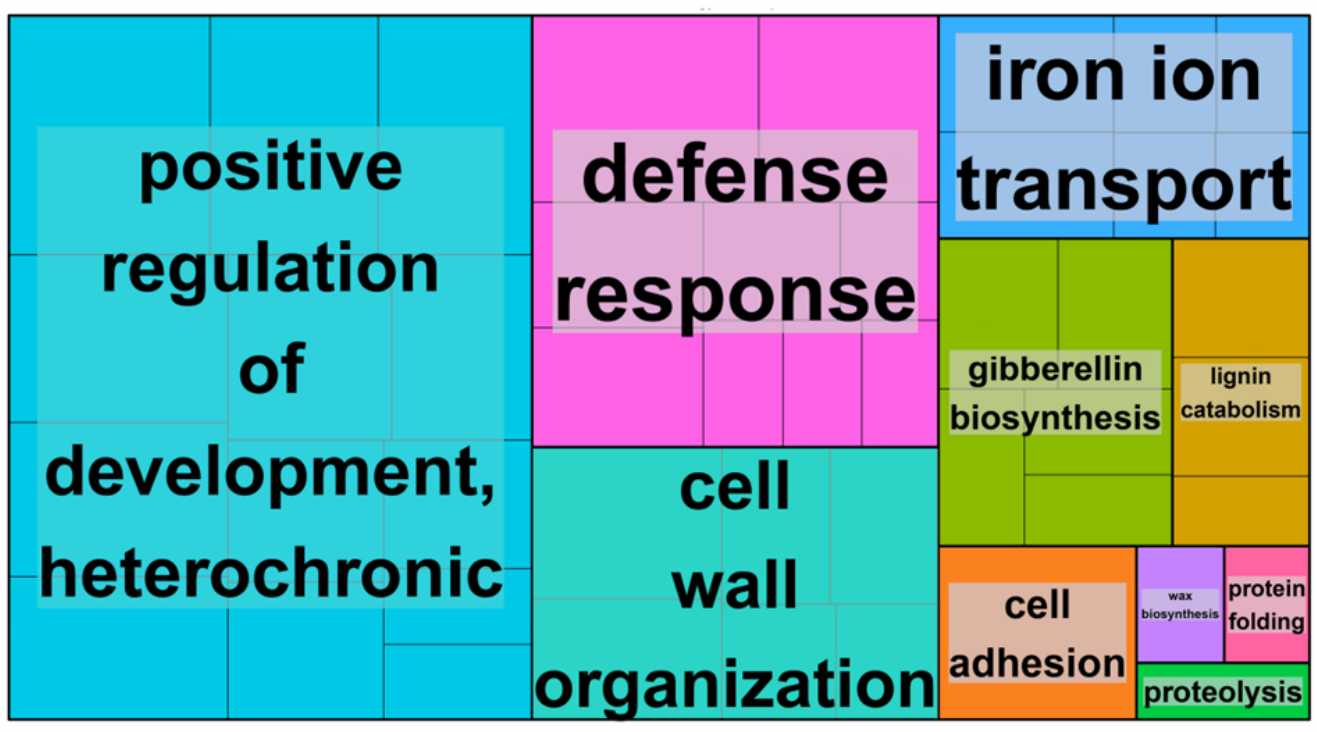

salt_stress

cold_stress

heat_stress

drought_stress

Figure 4. Soybean MTRMs under various abiotic stresses (a) Venn diagram of 37 kinds 
319 of soybean MTRMs under various abiotic stresses, including 14 drought-specific 320 MTRMs, seven salt-specific MTRMs, two heat-specific MTRMs, and two shared

321 MTRMs, (b) the miRNAs in the two shared MTRMs 31 and 493, and (c) a GO Treemap 322 of 37 MTRMs under abiotic stress.

Subsequently, we constructed the GO BP regulatory network of cooperative miRNAs 325 under soybean abiotic stress for the above main regulatory GO categories and miRNAs, 326 as shown in Figure 5(d). Gene expressions related to abiotic stress responses are mainly 327 regulated by multi-component miRNA families. For example, the miR167 family 328 regulates the resistance response pathway; the miR171 family regulates the gibberellin 329 biosynthesis pathway, while the miR395 family participates in regulating the iron uptake. 330 Moreover, some miRNAs have multiple GO functional partitions, such as miR156b, 331 which regulates developmental growth, the timing of developmental events, the response 332 to hormones, and the response to heavy metal cadmium. The miRNA families and 333 regulatory pathways involved in MTRM are detailed in Supplemental Dataset S4. 

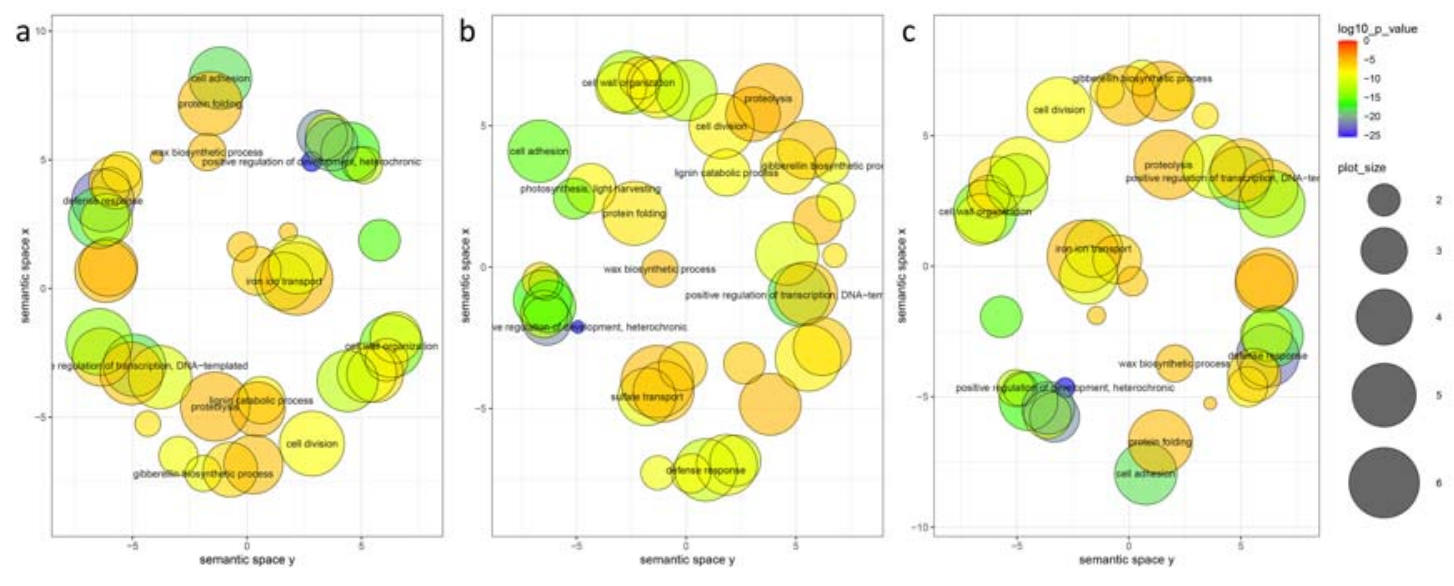

d

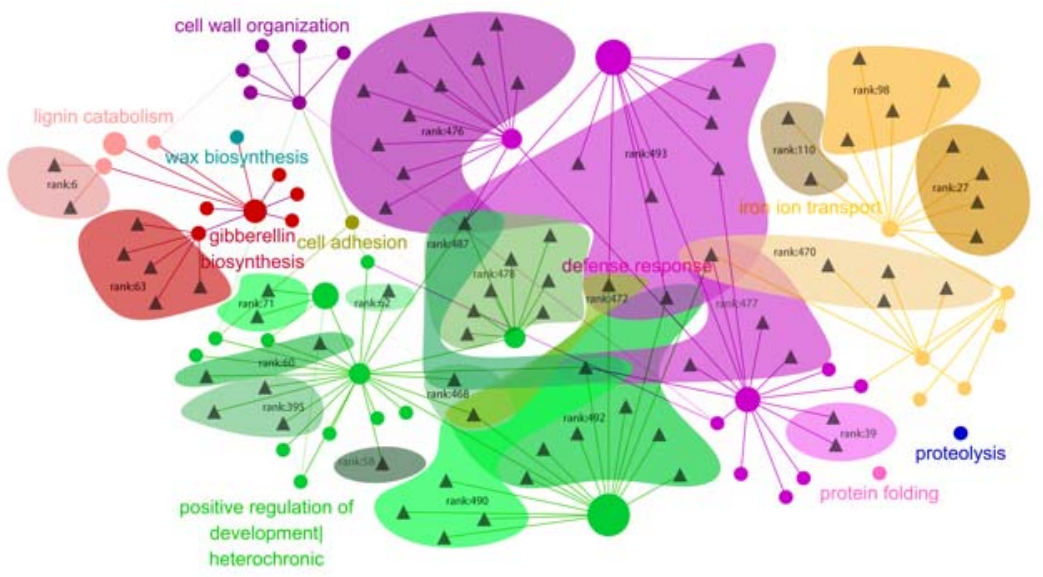

Figure 5. GO term analysis of MTRM genes under various abiotic stresses (a-c). (a) GO semantic correlation analysis of abiotic stress, (b) drought stress, and (c) salt stress, and (d) the GO BP regulatory network of cooperative miRNAs under abiotic stresses.

338 Triangles represent different miRNAs and circles represent different GOs. The size of the 339 circle is determined by the number of genes contained in the GO in this article. The color 340 of the circle depends on the representative GO. The areas with different colors show the 341 modules obtained by our method.

Discussions

miRNAs are major regulators of plant growth and development. They can also 
345 regulate environmental responses [58-67]. Hence, the study of the role of miRNAs is 346 crucial—not only to understanding the basic events of plant biology but to improve 347 breeding for higher yields and more resilient crop plants. While a variety of papers have 348 noted the role of one or a few miRNAs in regulating plant stress responses, a global 349 analysis of the cooperative interactions is lacking. To study miRNA regulation in 350 response to abiotic response in the soybean, we collected a large number of soybean 351 MTIs. In addition, we collected MTIs from the model plant Arabidopsis thaliana and the 352 legume Medicago truncatula and used them to predict potential targeting relationships in 353 soybeans through homologous gene analysis. Meanwhile, psRNAtarget, TAPIR, and 354 Targetfinder were used to support the targeting relationships. To ensure the reliability of 355 the data, we mainly chose drought stress, salt stress, cold stress, and heat stress.

In the process of bi-clustering soybean MTIs, CUBiBit obtained many MTRMs based on targeting relationships between miRNAs and their target genes. To merge related MTRMs, we proposed a multi-level iterative fusion method of soybean MTRMs based on soybean gene networks. The method determines whether the miRNAs of the two modules have intersections, and the distribution of their genes in the soybean gene network determines whether they can be fused into a unified class.

We mined 483 soybean MTRMs, which provide a data reference for the analysis of the cooperative miRNA mechanism of the soybean. Some MTRMs are involved in the biosynthesis of chalcone, which is derived from the general phenylpropanoid pathway 365 that plays a wide variety of roles in soybeans, as well as other plants. In most cases, gene 366 regulation in each MTRMs involved a multi-component miRNA gene family. In some 367 cases, these families were predicted to act cooperatively, which is consistent with the 368 conclusion of Wang et al. [27]. The functions of a specific miRNA family can represent 369 the main functions of miRNAs in the family, such as miR396 and its growth regulatory 370 factors (GRFs) [68]; moreover, these regulatory factors include drought stress, high salt 
371 stress, low-temperature stress, and ultraviolet radiation stress. Interestingly, we found that

372 miRNAs from different families are also involved in the same regulatory gene clusters,

373 which indicates that different miRNA families may have cross-family cooperative

374 regulatory mechanisms in regulating certain functions. In contrast, miRNAs in the same

375 family can be in different MTRMs; for example, the miR171 family (miR172b-5p, $376 \mathrm{miR} 172 \mathrm{~h}-5 \mathrm{p}, \mathrm{miR} 172 \mathrm{f}, \mathrm{miR} 172 \mathrm{~g}, \mathrm{miR} 172 \mathrm{j}$, and $\mathrm{miR} 172 \mathrm{k}$ ) are in multiple regulatory

377 modules during drought and salt stress. Such hub miRNAs may be useful research targets

378 for exploring soybean resistance mechanisms and resistance to breeding research under

379 different stresses. After further combining the analysis of differentially expressed genes

380 in soybeans under various stresses, we obtained the miRNA-GO regulatory network

381 under abiotic stress. The GO BP contains a variety of important related pathways for

382 understanding the common mechanisms in stress response. The research covering the

383 plant miRNA regulation module can analyze the coordination mechanism of miRNA

384 from a global perspective and determine the regulation relationship between modules,

385 which may help explore the regulation mechanism of soybean miRNAs. 


\section{Methods}

389 We collected soybean MTIs from Arabidopsis thaliana and Medicago truncatula 390 databases and publications on miRNAs and genes of soybean response to several abiotic 391 stresses. Subsequently, we used homology prediction on the collected MTIs to expand 392 the soybean MTIs. We used the biclustering method to mine the soybean MTRMs to 393 perform overlap analysis on the results and practiced redundancy removal. Then, based 394 on the soybean gene interaction network, biclustering was applied through multi-level 395 iteration. Finally, based on soybean abiotic stress-related miRNAs and genes, the fusion 396 regulatory module was screened to obtain soybean abiotic stress-related MTRMs. Figure 3976 shows a flowchart of our tasks and results.

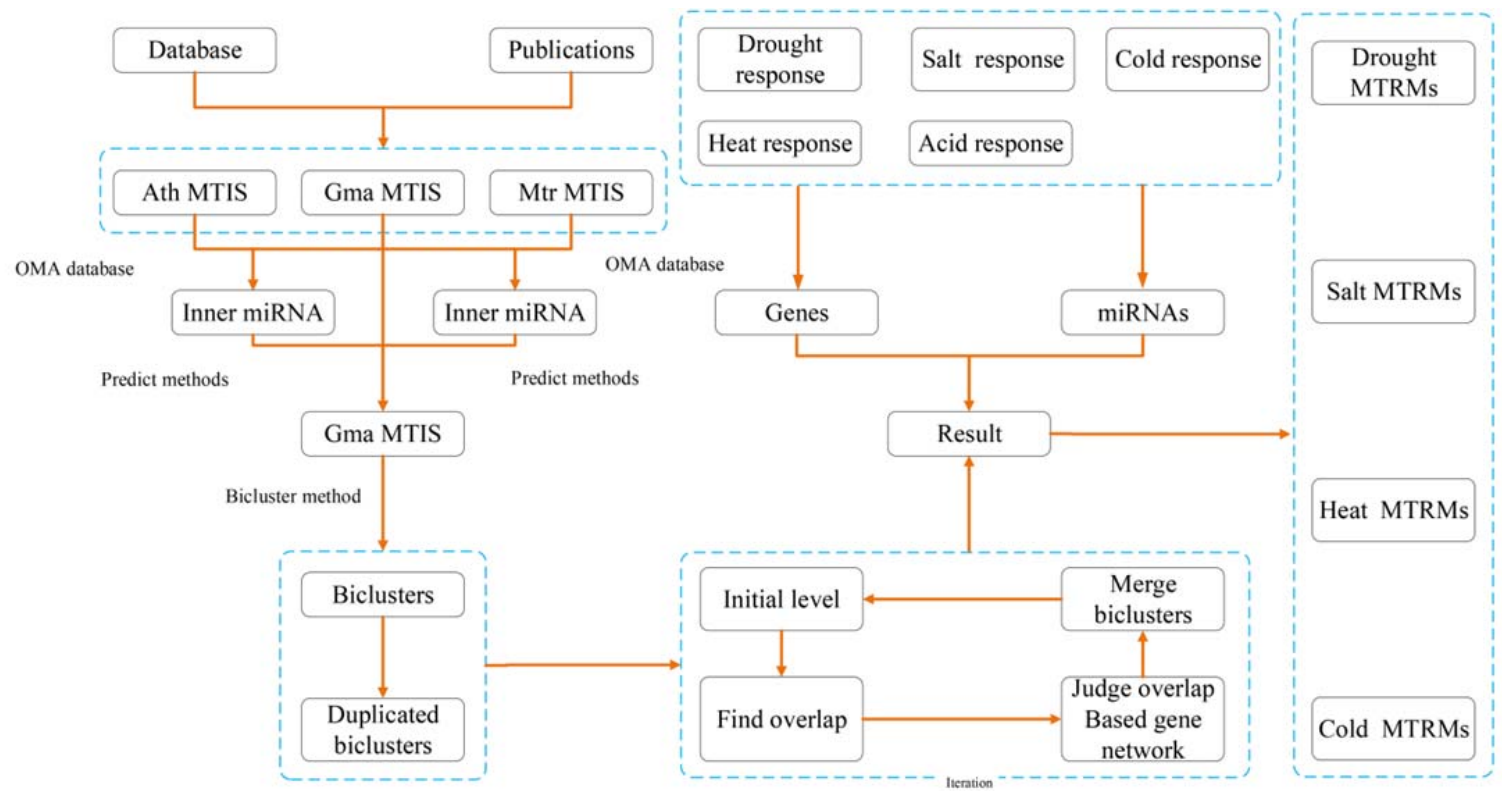

Figure 6. Flowchart of the authors' research method.

\section{Data collection}


404 databases (DPMIND, (c) Tarbase, mirTarbase, and Starbase [93-96]) and publications

405 (Supplemental Table 1). In addition, we collected the miRNA information of the three 406 species from the miRbase [97], the gene annotation of the species in the NCBI, 407 EnsemblPlants, and the Phytozome [98, 99]. We also downloaded the homologous genes 408 of Arabidopsis thaliana and Medicago truncatula in Orthologous MAtrix (OMA) [100]. 409 Besides, we downloaded the soybean cDNA sequence and soybean gene GO annotations 410 from SoyBase [101], and obtained soybean gene network data from SoyNet [102].

\section{Data processing}

414 Due to the differences in the annotation methods in various databases and the 415 differences in the data formats in the publications, we unified the miRNA and gene 416 formats in the data and put the data of the same species together. We annotated the 417 miRNA-target data based on the collected and processed miRNA details and the gene 418 annotations derived from the data of three species, including miRNA target data, related 419 notes and data sources. After processing the duplicated data, we obtained the 420 miRNA-target data of the three species.

\section{Homologous extension}

423 We chose Arabidopsis thaliana and Medicago truncatula to explore the potential 424 targets. Arabidopsis thaliana as a model plant has rich high-quality data. Medicago 425 truncatula and soybean are closely related and have many similar biological 426 characteristics. We extracted the miRNA sequence and removed redundant miRNAs with 427 the same sequence in the soybean and Arabidopsis thaliana. Subsequently, we extracted 
428 the target gene corresponding to the miRNA ID. Based on these targeted genes, we 429 obtained soybean genes homologous to these genes from the Arabidopsis 430 thaliana-soybean homologous genes downloaded by OMA. We assumed that if the 431 sequences coexist and the genes are homologous, targeting relationships may exist.

432 Therefore, these homologous genes may be targeted by these miRNAs in soybeans.

Targets obtained only based on homology information may not exist; so, we extracted these miRNA sequences and the cDNA sequence of target genes (SoyBase) and used miRNA-target prediction tools to predict potential relationships. Most of the

436 existing plant miRNA target gene prediction tools showed high accuracy in Arabidopsis

437 thaliana, but the prediction results in non-Arabidopsis thaliana plant data were different.

438 Therefore, we chose psRNAtarget, TAPIR, and Targetfinder, whose results were better in 439 non-Arabidopsis plants to predict potential soybean miRNA-target relationships [104].

440 The three prediction software tools have different scoring methods. We analyzed their 441 respective scores and merged them. The homology extension method for Medicago 442 truncatula-soybean is the same as above.

\section{Clustering method}

445 The current research on miRNA targeting relationships is mostly based on 446 one-to-one relative targeting. However, the miRNA targeting relationship is a complex 447 interaction. The traditional clustering method is to cluster the same type of data, such as 448 k-means, whose mining results in the miRNA-target regulatory module are poor because 449 the targeting of miRNAs is sparse. The relationship between miRNA and the target gene 450 is a bipartite graph structure; thus, the miRNA-target regulatory group can be found by 451 analyzing the bipartite graph. Bimax has been widely used in gene expression data [103]. 452 However, for more data, the calculation cost is higher. Domingo et al. proposed the BiBit 
453 [69], whose results are almost the same as that of Bimax, but the calculation time is still 454 longer. Subsequently, ParBiBit and CUBiBit were proposed [57], which shortened the 455 computing time and provided an optimized method for finding modules in larger data. 456 We added the miRNA-target data based on the homology expansion predictions obtained 457 from Arabidopsis thaliana and Medicago truncatula into the collected soybean 458 miRNA-target data and extracted the miRNA-Target data with GO annotations and 459 glyma2ID based on the soybean gene annotations of SoyBase. We used the CUBiBit to 460 perform bi-clustering to obtain the results.

\section{Overlap and iterative fusion}

The primary result obtained by CUBiBit was mostly a fully-connected bipartite graph. However, the relationship between miRNA and target gene is complex and interactive. Therefore, we proposed a method of iterative fusion for MTRM modules based on a gene interaction network (Figure 7).

We detected completely included classes in the clustering results and removed the 469 included classes as the initial level result. First, for each class of this level containing 470 miRNAs and genes, we judged the degree of overlap with other classes of miRNA and 471 genes to form alpha and beta matrices, both of which are upper triangular matrices. After

472 that, we set two thresholds of miRNA and genes that can be potentially merged for the 473 two classes. We then recorded the two classes that met the potential fusion class-class 474 table requirements to form a Boolean matrix. The initial threshold of alpha was 0.3, and 475 each iteration increased at a pace of 0.05 to conservatively determine the fusionable 476 module and to keep this value unchanged after increasing to 0.8 . It was sufficient if the 477 beta threshold was greater than 0 . Next, we extracted the union of two class genes, and 
478 the network blocks of this pair of genes with a depth of 2 layers based on the SoyNet

479 network, for each pair of classes in the potentially merged class-class table. Subsequently,

480 from the obtained block set, network blocks containing smaller classes were extracted.

481 We assumed that the network block with the most smaller class genes represented the

482 function of the genes in the smaller class. Therefore, judging the number of genes in the

483 major category of this network block can determine whether the genes of the two

484 categories are similar in function. If the genes of the two classes were concentrated on a

485 network block, which means that their genes interact closely and meet the conditions of

486 potential fusion, the two classes can be merged. We compared the number of genes in the

487 major category with the number of genes in all major categories in the sub-category

488 function module to obtain scores and determine the correlation. Finally, we compared the

489 number of genes in the major category with the number of genes in all major categories

490 in the sub-category function module to obtain scores to determine the correlation. The

491 threshold was recorded as gamma. When gamma $>0.3$ was satisfied, the two classes are

492 merged; otherwise, they would not be merged. For the class pairs that meet the fusion

493 condition, we arranged them in descending order of alpha value and performed top-down

494 non-repetitive fusion. Each class can only merge at most one class in one iteration. A new

495 class set was formed as the new level, and the fusion result was the output. The next

496 iteration would be performed and then another iteration until no fusion class pair could 497 meet the two conditions. 

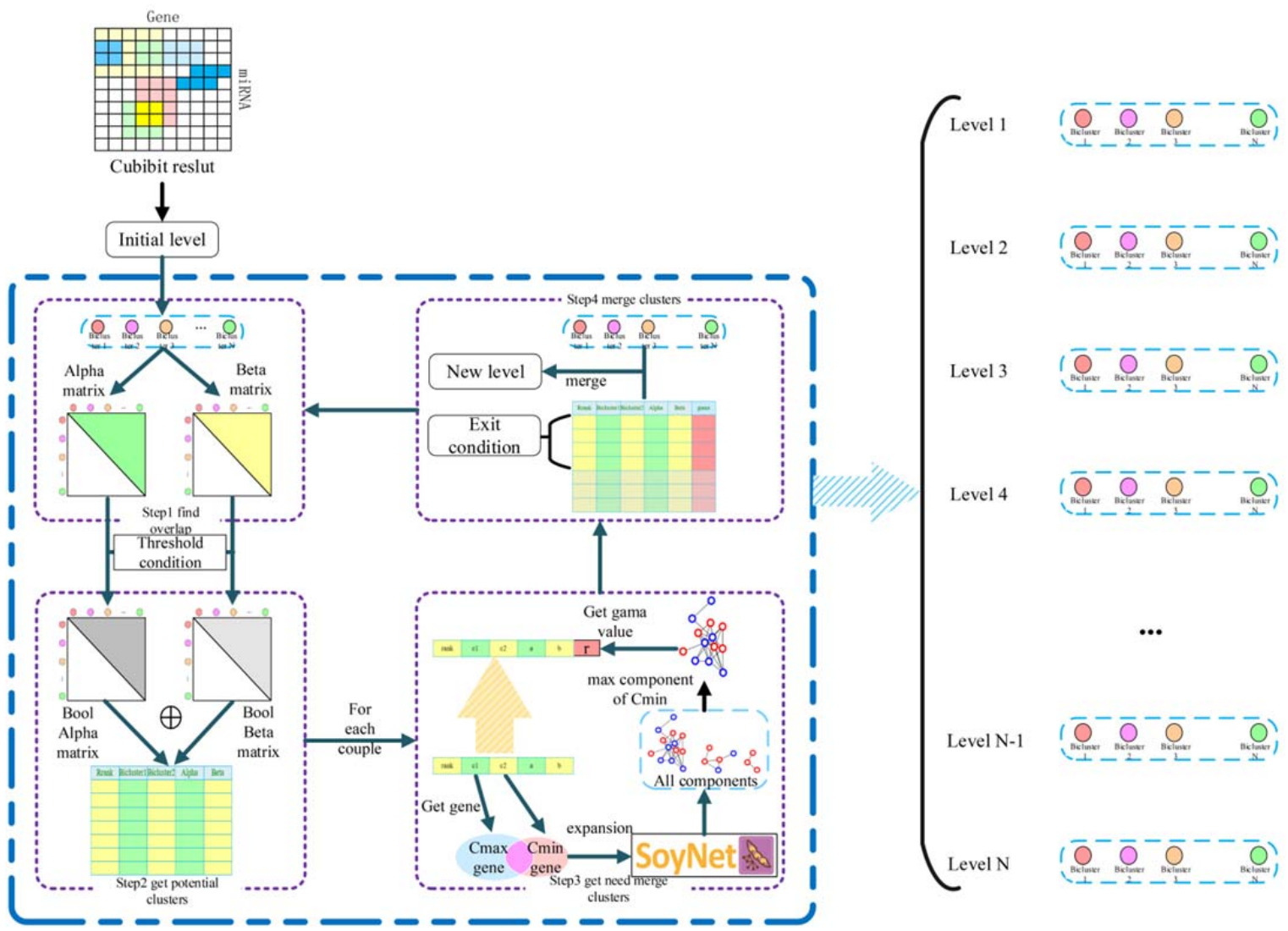

Figure 7. MTRM iterative merge algorithm flowchart used to derive a gene interaction network.

501 level were separately analyzed. For a bicluster, we extracted its genes, used SoyBase's GO BP and GO MF for enrichment analysis, and used the corrected GO ID with the smallest p-value as the best enrichment result for this type of cluster. When evaluating each class, only the smallest p-value was not enough to judge the importance of the class.

509 Here, we should observe the situation in all the GO IDs enriched by this class to make a reasonable judgment on this class. Therefore, we used the cluster score to evaluate the 
511 enrichment of the class. For all the enriched GO IDs of this class, we screened all the 512 results with a p-value of less than 0.05 and then used Eq. (1) to calculate the cluster score 513 of the class.

$$
\text { cluster score }=\frac{\sum_{1}^{n}\left(x_{i^{*}}-\log \left(\operatorname{correct} P_{i}\right)\right)}{\sum_{1}^{n} x_{i}}
$$

Among them, $n$ is the number of gene ontologies enriched in the module, $x_{i}$ is the number of genes enriched in the $i$-th GO, and correct $P_{i}$ is the adjusted p-value of the $517 \quad i$-th enriched GO.

\section{Abiotic Stress response MTRM}

We collected the miRNAs of soybeans that respond to drought, salt, acid, and low temperature based on our studies of publications [22, 48, 105-121]. At the same time, we collected the differentially expressed soybean genes under various stresses and screened these genes with foldchange $\geq 2$ and t-test p-value less than 0.05 as related genes under abiotic stress. Then we marked the genes in the module and calculated the p-value related to abiotic stress based on the hypergeometric distribution. Finally, we screened based on the cluster score calculated by the module, the p-value related to stress, and the proportion of miRNA related to stress. In addition, the screening procedures related to

528 drought and salt stress were consistent with the screening steps of the abiotic stress 529 module.

\section{Construction of miRNA-GO network under abiotic stress}

Based on the results of MTRM mining under stress, we first screened the GO of the enrichment results in the screened module by p-value to remove the GO with a p-value

534 less than $10^{-5}$; then, we performed a REVIGO semantic relevance analysis and extraction 
535 of concentrated representative GO channels. Based on the MTI data, the miRNA-GO 536 relationship data was constructed through the gene pointed to by the miRNA in the 537 module, and the enriched go pathway to which the gene belongs. The relationship 538 between GO is based on the results of REVIGO and the GO similarity calculation. The 539 relationship is presented by setting a threshold to remove some weaker relationships. 540 More detailed parameters are provided here or in the location of the specific figure.

Supplemental Table S1. Summary of ATH, GMA and MTR MTIs sources.

544 Supplemental Table S2. Potential targets found by homology expansion.

545 Supplemental Table S3. Comparison of the biclustering results of soybean MTRMs.

546 Supplemental Table S4. The top 5 GO enrichment of each top 5 soybean MTRMs.

547 Supplemental Table S5. Top 5 soybean abiotic response MTRMs.

548 Supplemental Dataset S1. miRNA function annotation.

549 Supplemental Dataset S2. Screening results of soybean MTRMs related to abiotic stress, 550 drought and salt stress.

551 Supplemental Dataset S3. Stress-specific MTRMs and shared MTRMs.

552 Supplemental Dataset S4. GO terms of miRNAs in the soybean abiotic response 553 MTRMs.

554 Supplemental Figure S1. Potential soybean MTIs predicted based on three prediction 555 tools. (a) 961 pairs of MTIs obtained based on the Arabidopsis data, (b) 986 pairs of 556 MTIs obtained based on the Medicago data, and (c) 1,189 soybean MTIs from the union 557 between (a) and (b). 
558 Supplemental Figure S2. The differentially expressed genes in each MTRM under other 559 stress after the screening.

$561 \quad$ Funding

562 This work was supported by the National Natural Science Foundation of China (grant no.

563 62072210), and the US National Science Foundation Plant Genome Program (grant 564 number \#IOS-1734145).

\section{Acknowledgments}

567 We would like to thank Ms. Carla Roberts for thoroughly proofreading this paper.

569 Conflict of interest statement. None declared.

570

571 


\section{Parsed Citations}

1. Ku, Y.S., Wong, J.W., Mui, Z, Liu, X., Hui, J.H., et al. (2015) Small RNAs in Plant Responses to Abiotic Stresses: Regulatory Roles and Study Methods. Int J Mol Sci, 16(10), 24532-24554.

Google Scholar: Author Only Title Only Author and Title

2. Li, S., Castillo-Gonzalez, C., Yu, B., Zhang, X. (2017) The functions of plant small RNAs in development and in stress responses.

Plant J, 90(4), 654-670.

Google Scholar: Author Only Title Only Author and Title

3. Ruiz-Ferrer, V., Voinnet, O. (2009) Roles of plant small RNAs in biotic stress responses. Annu Rev Plant Biol, 60, 485-510.

Google Scholar: Author Only Title Only Author and Title

4. Sunkar, R., Chinnusamy, V., Zhu, J., Zhu, J.K. (2007) Small RNAs as big players in plant abiotic stress responses and nutrient deprivation. Trends Plant Sci, 12(7), 301-309.

Google Scholar: Author Only Title Only Author and Title

5. Shukla, L.I., Chinnusamy, V., Sunkar, R. (2008) The role of microRNAs and other endogenous small RNAs in plant stress responses. Biochim Biophys Acta, 1779(11), 743-748.

Google Scholar: Author Only Title Only Author and Title

6. Song, X., Li, Y., Cao, X., Qi, Y. (2019) MicroRNAs and Their Regulatory Roles in Plant-Environment Interactions. Annu Rev Plant Biol, 70, 489-525.

Google Scholar: Author Only Title Only Author and Title

7. Sun, X., Lin, L., Sui, N. (2019) Regulation mechanism of microRNA in plant response to abiotic stress and breeding. Mol Biol Rep, 46(1), $1447-1457$.

Google Scholar: Author Only Title Only Author and Title

8. Hivrale, V., Zheng, Y., Puli, C.O.R., Jagadeeswaran, G., et al. (2016) Characterization of drought- and heat-responsive microRNAs in switchgrass. Plant Sci, 242, 214-223.

Google Scholar: Author Only Title Only Author and Title

9. Yu, Y., Ni, Z, Wang, Y., Wan, H., Hu, Z, et al. (2019) Overexpression of soybean miR169c confers increased drought stress sensitivity in transgenic Arabidopsis thaliana. Plant Sci, 285, 68-78.

Google Scholar: Author Only Title Only Author and Title

10. Pan, W.J., Tao, J.J., Cheng, T., Bian, X.H., Wei, W., et al. (2016) Soybean miR172a Improves Salt Tolerance and Can Function as a Long-Distance Signal. Mol Plant, 9(9), 1337-1340.

Google Scholar: Author Only Title Only Author and Title

11. Li, W., Wang, T., Zhang, Y., Li, Y. (2016) Overexpression of soybean miR172c confers tolerance to water deficit and salt stress, but increases ABA sensitivity in transgenic Arabidopsis thaliana. J Exp Bot, 67(1), 175-194.

Google Scholar: Author Only Title Only Author and Title

12. Zhou, Y., Liu, W., Li, X., Sun, D., Xu, K., et al. (2020) Integration of sRNA, degradome, transcriptome analysis and functional investigation reveals gma-miR398c negatively regulates drought tolerance via GmCSDs and GmCCS in transgenic Arabidopsis and soybean. BMC Plant Biol, 20(1), 190.

Google Scholar: Author Only Title Only Author and Title

13. Sun, Z, Wang, Y, Mou, F, Tian, Y, Chen, L, Zhang, S, Jiang, Q, Li, X (2015) Genome-Wide Small RNAAnalysis of Soybean Reveals Auxin-Responsive microRNAs that are Differentially Expressed in Response to Salt Stress in Root Apex. Front Plant Sci 6, 1273.

Google Scholar: Author Only Title Only Author and Title

14. Ning, L.H., Du,W.K., Song, H.N., Shao, H.B., Qi, W.C., et al. (2019) Identification of responsive miRNAs involved in combination stresses of phosphate starvation and salt stress in soybean root. Environmental and Experimental Botany, 167, 103823.

Google Scholar: Author Only Title Only Author and Title

15. Huang, S.C,. Lu, G.H., Tang, C.Y., Ji, Y.J., Tan, G.S., et al. (2018) Identification and comparative analysis of aluminum-induced microRNAs conferring plant tolerance to aluminum stress in soybean. Biologia Plantarum, 62(1), 97-108.

Google Scholar: Author Only Title Only Author and Title

16. Enright, AJ., John, B., Gaul, U., Tuschl, T., Sander, C., Marks, D.S. (2003) MicroRNAtargets in Drosophila. Genome Biol, $5(1)$, R1. Google Scholar: Author Only Title Only Author and Title

17. Azra, K., Dominic, G., Matthew, N.P., Rachel, W., Lauren, R., et al. (2005) Combinatorial microRNA target predictions. Nat Genet, 37(5), 495-500.

Google Scholar: Author Only Title Only Author and Title

18. Wahdan-Alaswad, R., Liu, B. (2013) "Sister" miRNAs in cancers. Cell Cycle, 12(24),3703-3704.

Google Scholar: Author Only Title Only Author and Title

19. Wang, S., Huang, J., Lyu, H., Lee, C.K., Tan, J., Wang, J., Liu, B. (2013) Functional cooperation of miR-125a, miR-125b, and miR-205 in 
entinostat-induced downregulation of erbB2/erbB3 and apoptosis in breast cancer cells. Cell Death Dis, 4, e556.

Google Scholar: Author Only Title Only Author and Title

20. Ivanovska l., Cleary M.A (2009) Combinatorial microRNAs: working together to make a difference, 7(20), 3137-3142.

Google Scholar: Author Only Title Only Author and Title

21. Frampton, AE., Castellano, L., Colombo, T., Giovannetti, E., Krell, J., et al. (2014) MicroRNAs cooperatively inhibit a network of tumor suppressor genes to promote pancreatic tumor growth and progression. Gastroenterology, 146(1), 268-277 e218.

Google Scholar: Author Only Title Only Author and Title

22. Ramanjulu, S., Li, Y.F., Guru, J. (2012) Functions of microRNAs in plant stress responses. Trends in plant science, 17(4), 196-203. Google Scholar: Author Only Title Only Author and Title

23. Xu, Y., Guo, M., Liu, X., Wang, C., Liu, Y. (2014) Inferring the soybean (Glycine max) microRNAfunctional network based on target gene network. Bioinformatics, 30(1), 94-103.

Google Scholar: Author Only Title Only Author and Title

24. Yoshikawa, T., Ozawa, S., Sentoku, N., Itoh, J., Nagato, Y., Yokoi, S. (2013) Change of shoot architecture during juvenile-to-adult phase transition in soybean. Planta, 238(1), 229-237.

Google Scholar: Author Only Title Only Author and Title

25. Wang, T., Sun, M.Y., Wang, X.S., Li, W.B., Li, Y.G. (2016) Over-Expression of GmGla-Regulated Soybean miR172a Confers Early Flowering in Transgenic Arabidopsis thaliana. Int J Mol Sci, 17(5).

Google Scholar: Author Only Title Only Author and Title

26. Li, H., Deng, Y., Wu, T., Subramanian, S., Yu, O. (2010) Misexpression of miR482, miR1512, and miR1515 increases soybean nodulation. Plant Physiol, 153(4), 1759-1770.

Google Scholar: Author Only Title Only Author and Title

27. Wang, R., Yang, Z, Fei, Y., Feng, J., Zhu, H., et al. (2019) Construction and analysis of degradome-dependent microRNA regulatory networks in soybean. BMC Genomics, 20(1), 534.

Google Scholar: Author Only Title Only Author and Title

28. Shalgi, R., Lieber, D., Oren, M., Pilpel, Y. (2007) Global and local architecture of the mammalian microRNAtranscription factor regulatory network. PLoS Comput Biol, 3(7), e131.

Google Scholar: Author Only Title Only Author and Title

29. Xu, J., Li, C.X., Li, Y.S., Lv, J.Y., Ma, Y., et al. (2011) MiRNA-miRNA synergistic network: construction via co-regulating functional modules and disease miRNA topological features. Nucleic Acids Res, 39(3), 825-836.

Google Scholar: Author Only Title Only Author and Title

30. Xie, J., Ma, A, Fennell, A, Ma, Q., Zhao, J. (2019) It is time to apply biclustering: a comprehensive review of biclustering applications in biological and biomedical data. Brief Bioinform, 20(4), 1449-1464.

Google Scholar: Author Only Title Only Author and Title

31. Tanay, A, Sharan, R., Shamir, R. (2002) Discovering statistically significant biclusters in gene expression data. Bioinformatics, 18 Suppl 1, S136-144.

Google Scholar: Author Only Title Only Author and Title

32. Bergmann, S., Ihmels, J., Barkai, N. (2003) Iterative signature algorithm for the analysis of large-scale gene expression data. Phys Rev E Stat Nonlin Soft Matter Phys, 67(3 Pt 1), 031902.

Google Scholar: Author Only Title Only Author and Title

33. Prelic, A, Bleuler. S., Zmmermann, P., Wille, A, Buhlmann, P., et al. (2006) Asystematic comparison and evaluation of biclustering methods for gene expression data. Bioinformatics, 22(9), 1122-1129.

Google Scholar: Author Only Title Only Author and Title

34. Li, G., Ma, Q., Tang, H., Paterson, AH., Xu, Y. (2009) QUBIC: a qualitative biclustering algorithm for analyses of gene expression data. Nucleic Acids Res, 37(15), e101.

Google Scholar: Author Only Title Only Author and Title

35. Hochreiter, S., Bodenhofer, U., Heusel, M., Mayr, A, Mitterecker, A, et al. (2010) FABIA: factor analysis for bicluster acquisition.

Bioinformatics, 26(12), 1520-1527.

Google Scholar: Author Only Title Only Author and Title

36. Medina, I., Carbonell, J., Pulido, L., Madeira, S.C., Goetz, S., et al. (2010) Babelomics: an integrative platform for the analysis of transcriptomics, proteomics and genomic data with advanced functional profiling. Nucleic Acids Res, 38(Web Server issue), W210-213.

Google Scholar: Author Only Title Only Author and Title

37. Goncalves, J.P., Madeira, S.C., Oliveira, AL. (2009) BiGGEsTS: integrated environment for biclustering analysis of time series gene expression data. BMC Res Notes, 2, 124.

Google Scholar: Author Only Title Only Author and Title

38. Madeira, S.C., Teixeira, M.C., Sa-Correia, I., Oliveira, AL. (2010) Identification of regulatory modules in time series gene expression 
data using a linear time biclustering algorithm. IEEE/ACM Trans Comput Biol Bioinform, 7(1), 153-165.

Google Scholar: Author Only Title Only Author and Title

39. Goncalves, J.P., Madeira, S.C. (2014) LateBiclustering: Efficient Heuristic Agorithm for Time-Lagged Bicluster Identification. IEEE/ACM Trans Comput Biol Bioinform, 11(5), 801-813.

Google Scholar: Author Only Title Only Author and Title

40. Henriques, R., Ferreira, F.L., Madeira, S.C. (2017) BicPAMS: software for biological data analysis with pattern-based biclustering. BMC Bioinformatics, 18(1), 82.

Google Scholar: Author Only Title Only Author and Title

41. Henriques, R., Madeira, S.C. (2014) BicPAM: Pattern-based biclustering for biomedical data analysis. Algorithms Mol Biol, 9(1), 27. Google Scholar: Author Only Title Only Author and Title

42. Henriques, R., Madeira, S.C. (2016) BicNET: Flexible module discovery in large-scale biological networks using biclustering. Algorithms Mol Biol, 11, 14.

Google Scholar: Author Only Title Only Author and Title

43. Bentham, R.B., Bryson, K., Szabadkai, G. (2017) MCbiclust: a novel algorithm to discover large-scale functionally related gene sets from massive transcriptomics data collections. Nucleic Acids Res, 45(15), 8712-8730.

Google Scholar: Author Only Title Only Author and Title

44. Caldas, J., Kaski, S. (2011) Hierarchical generative biclustering for microRNAexpression analysis. J Comput Biol, 18(3), $251-261$. Google Scholar: Author Only Title Only Author and Title

45. Yoon, S., De, M.G. (2005) Prediction of regulatory modules comprising microRNAs and target genes. Bioinformatics, 21(Suppl 2), ii93-100.

Google Scholar: Author Only Title Only Author and Title

46. Huang, J.Y., Brutlag, D.L. (2001) The EMOTIF database. Nucleic Acids Res, 29(1), 202-204.

Google Scholar: Author Only Title Only Author and Title

47. Pio, G., Ceci, M., D'Elia, D., Loglisci, C., Malerba, D. (2013) A novel biclustering algorithm for the discovery of meaningful biological correlations between microRNAs and their target genes. BMC Bioinformatics, 14(Suppl 7), S8.

Google Scholar: Author Only Title Only Author and Title

48. Muhammad, D., Muhammad Y.K.B., Iftekhar AB. (2016) Profiling and annotation of microRNAs and their putative target genes in chilli (Capsicum annuum L.) using ESTs. Gene Reports, 5, 62-69.

Google Scholar: Author Only Title Only Author and Title

49. Xu, Y.G., Guo, M.Z, Liu, X.Y., Wang, C.Y., Liu, Y. (2014) SoyFN: a knowledge database of soybean functional networks. Database, 2014(1), bau019.

Google Scholar: Author Only Title Only Author and Title

50. Fei, Y., Wang, R., Li, H., Liu, S., Zhang, H., Huang, J. (2018) DPMIND: degradome-based plant miRNA-target interaction and network database. Bioinformatics, 34(9), 1618-1620.

Google Scholar: Author Only Title Only Author and Title

51. Yang, J.H., Li, J.H., Shao, P., Zhou, H., Chen, Y.Q., Qu, L.H. (2011) starBase: a database for exploring microRNAmRNA interaction maps from Argonaute CLIP-Seq and Degradome-Seq data. Nucleic Acids Res, 39(Database issue), D202-209.

Google Scholar: Author Only Title Only Author and Title

52. Sethupathy, P., Corda, B., Hatzigeorgiou, AG. (2006) TarBase: Acomprehensive database of experimentally supported animal microRNA targets. RNA, 12(2), 192-197.

Google Scholar: Author Only Title Only Author and Title

53. Dai, X., Zhuang, Z, Zhao, P.X. (2018) psRNATarget: a plant small RNAtarget analysis server (2017 release). Nucleic Acids Res, 46(W1), W49-W54.

Google Scholar: Author Only Title Only Author and Title

54. Dai, X., Zhao, P.X. (2011) psRNATarget: a plant small RNA target analysis server. Nucleic Acids Res, 39(Web Server issue), W155159.

Google Scholar: Author Only Title Only Author and Title

55. Bonnet, E., He, Y., Billiau, K., Van, de, P.Y. (2010) TAPIR, a web server for the prediction of plant microRNA targets, including target mimics. Bioinformatics, 26(12), 1566-1568.

Google Scholar: Author Only Title Only Author and Title

56. Bo, X.C., Wang, S.Q. (2005) TargetFinder: a software for antisense oligonucleotide target site selection based on MAST and secondary structures of target mRNABioinformatics, 21(8), 1401-1402.

Google Scholar: Author Only Title Only Author and Title

57. JorgeGonzález-Domínguez, R.R.E. (2019) Accelerating binary biclustering on platforms with CUDA-enabled GPUs. Information Sciences, 496, 317-325. 
Google Scholar: Author Only Title Only Author and Title

58. Khraiwesh, B., Zhu, J.K., Zhu, J. (2012) Role of miRNAs and siRNAs in biotic and abiotic stress responses of plants. Biochim Biophys Acta, 1819(2), 137-148.

Google Scholar: Author Only Title Only Author and Title

59. Kulcheski, F.R., Molina, L.G., da, Fonseca, G.C., de, Morais, G.L., de, Oliveira, L.F., Margis, R. (2016) Novel and conserved microRNAs in soybean floral whorls. Gen, 575(2 Pt 1), 213-223.

Google Scholar: Author Only Title Only Author and Title

60. Zhu, Q.H., Helliwell, C.A (2011) Regulation of flowering time and floral patterning by miR172. J Exp Bot, 62(2), 487-495.

Google Scholar: Author Only Title Only Author and Title

61. Chen, X. (2001) A microRNAas a translational repressor of APETALA2 in Arabidopsis flower development. Science, 303(5666), 20222025.

Google Scholar: Author Only Title Only Author and Title

62. Aukerman, M.J., Sakai, H. (2003) Regulation of flowering time and floral organ identity by a MicroRNAand its APETALA2-like target genes. Plant Cell, 15(11), 2730-2741.

Google Scholar: Author Only Title Only Author and Title

63. Wong, J., Gao, L., Yang, Y., Zhai, J., Arikit, S., et al. (2014) Roles of small RNAs in soybean defense against Phytophthora sojae infection. Plant J, 79(6), 928-940.

Google Scholar: Author Only Title Only Author and Title

64. Mao, G., Turner, M., Yu, O., Subramanian, S. (2013) miR393 and miR164 influence indeterminate but not determinate nodule development. Plant Signal Behav, 8(10), doi: 10 4161/psb 26753.

Google Scholar: Author Only Title Only Author and Title

65. Turner, M., Nizampatnam, N.R., Baron, M., Coppin, S., Damodaran, S., et al. (2013) Ectopic expression of miR160 results in auxin hypersensitivity, cytokinin hyposensitivity, and inhibition of symbiotic nodule development in soybean. Plant Physiol, 162(4), $2042-2055$.

Google Scholar: Author Only Title Only Author and Title

66. Wang, Y., Li, K., Chen, L., Zou Y., Liu, H., et al. (2015) MicroRNA167-Directed Regulation of the Auxin Response Factors GmARF8a and GmARF8b Is Required for Soybean Nodulation and Lateral Root Development. Plant Physiol, 168(3), 984-999.

Google Scholar: Author Only Title Only Author and Title

67. Yan, Z, Hossain, M.S., Wang, J., Valdes-Lopez, O., Liang, Y., et al. (2013) miR172 regulates soybean nodulation. Mol Plant Microbe Interact, 26(12), 1371-1377.

Google Scholar: Author Only Title Only Author and Title

68. Omidbakhshfard, M.A, Proost, S., Fujikura, U., Mueller-Roeber, B. (2015) Growth-Regulating Factors (GRFs): ASmall Transcription Factor Family with Important Functions in Plant Biology. Mol Plant, 8(7), 998-1010.

Google Scholar: Author Only Title Only Author and Title

69. Rodriguez-Baena, D.S., Perez-Pulido, AJ., Aguilar-Ruiz, J.S. (2011) A biclustering algorithm for extracting bit-patterns from binary datasets. Bioinformatics, 27(19), 2738-2745.

Google Scholar: Author Only Title Only Author and Title

70. Yan, Z, Hossain, M., Arikit, S., et al. (2015) Identification of micro RNAs and their mRNAtargets during soybean nodule development: functional analysis of the role of miR393j-3p in soybean nodulation. New Phytologist, 207(3), 748-759.

Google Scholar: Author Only Title Only Author and Title

71. Yan, Z, Hossain, M., Lopez, O., et al. (2016) Identification and functional characterization of soybean root hair microRNAs expressed in response to Bradyrhizobium japonicum infection. Plant Biotechnology Journal, 14(1), 332-341.

Google Scholar: Author Only Title Only Author and Title

72. Liu, T., Fang, C., Ma, Y., et al. (2016) Global investigation of the co-evolution of MIRNAgenes and micro RNA targets during soybean domestication. Plant Journal, 85(3), 396-409.

Google Scholar: Author Only Title Only Author and Title

73. Ding, X., Li, J., Zhang, H., et al. (2016) Identification of miRNAs and their targets by high-throughput sequencing and degradome analysis in cytoplasmic male-sterile line NJCMS1A and its maintainer NJCMS1B of soybean. BMC Genomics, $17,24$.

Google Scholar: Author Only Title Only Author and Title

74. Chen, H., Adam, Arsovski, A, Yu, K., et al. (2017) Deep sequencing leads to the identification of eukaryotic translation initiation factor $5 A$ as a key element in Rsv1-mediated lethal systemic hypersensitive response to Soybean mosaic virus infection in soybean.

Molecular Plant Pathology, 18(3), 391-404.

Google Scholar: Author Only Title Only Author and Title

75. Xu, S., Liu, N., Mao, W., et al. (2016) Identification of chilling-responsive microRNAs and their targets in vegetable soybean (Glycine max L.). SCIENTIFIC REPORTS, 6.

Google Scholar: Author Only Title Only Author and Title 
76. Chen, H., Arsovski, AA, Yu, K., et al. (2017) Correction: Genome-Wide Investigation Using sRNA-Seq, Degradome-Seq and Transcriptome-Seq Reveals Regulatory Networks of microRNAs and Their Target Genes in Soybean during Soybean mosaic virus Infection. PLoS ONE, 12(11), 1.

Google Scholar: Author Only Title Only Author and Title

77. Turner, M., Yu, O., Subramanian, S. (2012) Genome organization and characteristics of soybean microRNAs. BMC Genomics, 13(1), 169.

Google Scholar: Author Only Title Only Author and Title

78. Shamimuzzaman, M., Vodkin, L. (2012) Identification of soybean seed developmental stage-specific and tissue-specific miRNA targets by degradome sequencing. BMC Genomics, 13(1).

Google Scholar: Author Only Title Only Author and Title

79. Xu, F., Liu, Q., Chen, L., et al. (2013) Genome-wide identification of soybean microRNAs and their targets reveals their organspecificity and responses to phosphate starvation. BMC Genomics, 14(1), 66.

Google Scholar: Author Only Title Only Author and Title

80. Fang, X., Zhao, Y., Ma, Q., et al. (2013) Identification and comparative analysis of cadmium tolerance- associated miRNAs and their targets in two soybean genotypes. Plos One, 8(12), e81471.

Google Scholar: Author Only Title Only Author and Title

81. Xu, M., Li, Y., Zhang, Q., et al. (2014) Novel MiRNA and PhasiRNABiogenesis Networks in Soybean Roots from Two Sister Lines That Are Resistant and Susceptible to SCN Race 4. PLoS ONE, 9(10), 1-20.

Google Scholar: Author Only Title Only Author and Title

82. Ye, C., Xu, H., Shen, E., et al. (2014) Genome-wide identification of non-coding RNAs interacted with microRNAs in soybean. FRONTIERS IN PLANT SCIENCE, 5, 743.

Google Scholar: Author Only Title Only Author and Title

83. Song, Q., Liu, Y., Hu, X., et al. (2011) Identification of miRNAs and their target genes in developing soybean seeds by deep sequencing. BMC PLANT BIOLOGY, 11(1), 1-16.

Google Scholar: Author Only Title Only Author and Title

84. Xu, M., Zhang, L., Li, W., et al. (2014) Stress-induced early flowering is mediated by miR169 in Arabidopsis thaliana. Journal of Experimental Botany, 65(1), 89-101.

Google Scholar: Author Only Title Only Author and Title

85. Ding, J., Li, D., Ohler, U., et al. (2012) Genome-wide search for miRNA-target interactions in Arabidopsis thaliana with an integrated approach. BMC Genomics, 13(Suppl 3), S3.

Google Scholar: Author Only Title Only Author and Title

86. German, M.A, Pillay, M., Jeong, D-H. (2008) Global identification of microRNA-target RNA pairs by parallel analysis of RNAends. Nature biotechnology, 26(8), 941-946.

Google Scholar: Author Only Title Only Author and Title

87. Addo-Quaye, C., Eshoo, T.W., Bartel, D.P., et al. (2008) Endogenous siRNAand miRNA targets identified by sequencing of the Arabidopsis degradome. Current biology: CB, 18(10), 758-762.

Google Scholar: Author Only Title Only Author and Title

88. Ma, X., Yu, D., Shao, W., et al. (2018) Transcriptome-wide identification and characterization of the copper and cadmium stressresponsive small RNAs and their targets in Arabidopsis thaliana. Plant \& Soil, 429(1-2), 391-405.

Google Scholar: Author Only Title Only Author and Title

89. Lauressergues, D., Delaux, P.M., Formey, D., et al. (2012) The microRNAmiR171h modulates arbuscular mycorrhizal colonization of Medicago truncatula by targeting NSP2. Plant J, 72(3): 512-522.

Google Scholar: Author Only Title Only Author and Title

90. Zhou, Z, Zeng, H., Liu, Z, et al. (2012) Genome-wide identification of Medicago truncatula microRNAs and their targets reveals their differential regulation by heavy metal. Plant Cell Environ, 35(1), 86-99.

Google Scholar: Author Only Title Only Author and Title

91. Devers, E.A, Branscheid, A, May, P. (2011) Stars and Symbiosis: MicroRNA and MicroRNA*-Mediated Transcript Cleavage Involved in Arbuscular Mycorrhizal Symbiosis. PLANT PHYSIOLOGY, 156(4), 1990-2010.

Google Scholar: Author Only Title Only Author and Title

92. Senthil, S., Gary, S., Oliver, Y. (2006) Endogenous isoflavones are essential for the establishment of symbiosis between soybean and Bradyrhizobium japonicum. The Plant journal : for cell and molecular biology, 48(2), 261-273.

Google Scholar: Author Only Title Only Author and Title

93. Fei, Y.H., Wang, R., Li, H.Y., Liu, S., Zhang, H.S., Huang, J. (2018) DPMIND: degradome-based plant miRNA-target interaction and network database. Bioinformatics, 34(9), 1618-1620.

Google Scholar: Author Only Title Only Author and Title

94. Vachos, I.S., Paraskevopoulou, M.D., Karagkouni, D., Georgakilas, G., Vergoulis, T., et al. (2015) DIANA-TarBase v7.0: indexing 
more than half a million experimentally supported miRNA:mRNĀinteractions. N̄ucleic Acids Rèsearch, 43, D153-D159.

Google Scholar: Author Only Title Only Author and Title

95. Hsu, S.D., Lin, F.M., Wu, W.Y., Liang, C., Huang, W.C., et al. (2011) miRTarBase: a database curates experimentally validated microRNA-target interactions. Nucleic Acids Research, 39, D163-D169.

Google Scholar: Author Only Title Only Author and Title

96. Li, J.H., Liu, S., Zhou, H., Qu, L.H., Yang, J.H. (2014) starBase v2.0: decoding miRNA-ceRNA, miRNAncRNAand protein-RNA interaction networks from large-scale CLIP-Seq data. NUCLEIC ACIDS RESEARCH, 42, D92-D97.

Google Scholar: Author Only Title Only Author and Title

97. Harpreet, K.S., Stijn, V.D., Anton, J.E. (2007) miRBase: tools for microRNAgenomics. Nucleic Acids Research, 36(50), 5.

Google Scholar: Author Only Title Only Author and Title

98. Howe, K.L., Contreras-Moreira, B., De, Silva, N., Maslen, G., Akanni, W., et al. (2019) Ensembl Genomes 2020-enabling nonvertebrate genomic research. Nucleic Acids Research, 48, D689-D695.

Google Scholar: Author Only Title Only Author and Title

99. Goodstein, D.M., Shu, S., Howson, R., Neupane, R., Hayes, R.D., et al. (2012) Phytozome: a comparative platform for green plant genomics. Nucleic Acids Research, 40, D1178-D1186.

Google Scholar: Author Only Title Only Author and Title

100. Adrian, M.A, Adrian, S., Gaston, H.G., Christophe, D. (2011) OMA2011: orthology inference among 1000 complete genomes. Nucleic Acids Research, 39, D289-D294.

Google Scholar: Author Only Title Only Author and Title

101. Grant, D., Nelson, R.T., Cannon, S.B., Shoemaker, R.C. (2010) SoyBase, the USDAARS soybean genetics and genomics database. Nucleic Acids Research, 38, D843-D846.

Google Scholar: Author Only Title Only Author and Title

102. Eiru, K., Sohyun, H., Insuk, L. (2017) SoyNet: a database of co-functional networks for soybean Glycine max. Nucleic Acids Research, 45, D1082-D1089.

Google Scholar: Author Only Title Only Author and Title

103. Amela, P., Stefan, B., Philip, Z, Anja, W., Peter, B., et al. (2006) Asystematic comparison and evaluation of biclustering methods for gene expression data. Bioinformatics, 22(9), 1122-1129.

Google Scholar: Author Only Title Only Author and Title

104. Prashant, K.S., Taraka, R.M., Priyanka, P., lan, T.B., Shree, P.P. (2014) A comparison of performance of plant miRNA target prediction tools and the characterization of features for genome-wide target prediction. BMC genomics, $15(1), 348$.

Google Scholar: Author Only Title Only Author and Title

105. Zhang, ZB., Dunwell, J.M., Zhang, Y.M. (2018) An integrated omics analysis reveals molecular mechanisms that are associated with differences in seed oil content between Glycine max and Brassica napus. BMC Plant Biology, 18(1), 1-15.

Google Scholar: Author Only Title Only Author and Title

106. Ning, L.H., Du, W.k., Song, H.N., Shao, H.B., Qi, W.C., et al. (2019) Identification of responsive miRNAs involved in combination stresses of phosphate starvation and salt stress in soybean root. Environmental and Experimental Botany, 167, 103823.

Google Scholar: Author Only Title Only Author and Title

107. Hélène, P., Jérémy, M., Martin, C., Caroline, H., Christine, L.B. (2019) Small RNA diversity and roles in model legumes. The Model Legume Medicago truncatula, 948-962.

Google Scholar: Author Only Title Only Author and Title

108. Gupta, O.P., Dahuja, A, Sachdev, A, Kumari, S., Jain, P.K., et al. (2019) Conserved miRNAs modulate the expression of potential transcription factors of isoflavonoid biosynthetic pathway in soybean seeds. Molecular Biology Reports, 46(4), 3713-3730.

Google Scholar: Author Only Title Only Author and Title

109. Sha, AH., Chen, Y.H., Ba, H.P., Shan, ZH., Zhang, X.J., et al. (2012) Identification of Glycine Max MicroRNAs in response to phosphorus deficiency. Journal of Plant Biology, 55(4), 268-280.

Google Scholar: Author Only Title Only Author and Title

110. Yu, J.Y., Zhang, ZG., Huang, S.Y., Han, X., Wang, X.Y., et al. (2019) Analysis of miRNAs Targeted Storage Regulatory Genes during Soybean Seed Development Based on Transcriptome Sequencing. Genes, 10(6), 408.

Google Scholar: Author Only Title Only Author and Title

111. Chen, L.F., Ding, X.L., Zhang, H., He, T.T., Li, Y.W., et al. (2018) Comparative analysis of circular RNAs between soybean cytoplasmic male-sterile line NJCMS1A and its maintainer NJCMS1B by high-throughput sequencing. BMC Genomics, $19(1), 1-14$.

Google Scholar: Author Only Title Only Author and Title

112. Li, HY, Dong, YY, Yin, HL, Wang, N, Yang, J, et al. (2011) Characterization of the stress associated microRNAs in Glycine max by deep sequencing BMC PLANT BIOLOGY 11(1), 170-181.

Google Scholar: Author Only Title Only Author and Title 
113. Senthil, S. (2012) microRNA Regulation of Symbiotic Nodule Development in Legumes. MicroRNAs in Plant Development and Stress Responses, 15, 177-195.

Google Scholar: Author Only Title Only Author and Title

114. Sun, ZX., Wang, Y.N., Mou, F.P., Tian, Y.P., et al. (2016) Genome-Wide Small RNAAnalysis of Soybean Reveals Auxin-Responsive microRNAs that are Differentially Expressed in Response to Salt Stress in Root Apex. FRONTIERS IN PLANT SCIENCE, 6, 1273.

Google Scholar: Author Only Title Only Author and Title

115. Zheng, Y., Hivrale, V., Zhang, X., Valliyodan, B., Lelandais-Briere, C., et al. (2016) Small RNA profiles in soybean primary root tips under water deficit. BMC systems biology, 10, 126.

Google Scholar: Author Only Title Only Author and Title

116. Xu, S.C, Liu, N., Mao, W.H., Hu, Q.Z, Wang, G.F., Gong, Y.M. (2016) Identification of chilling-responsive microRNAs and their targets in vegetable soybean (Glycine max L.). SCIENTIFIC REPORTS, 6.

Google Scholar: Author Only Title Only Author and Title

117. Dong, ZH., Shi, L., Wang, Y.W., Chen, L., Cai, ZM., et al. (2013) Identification and Dynamic Regulation of microRNAs Involved in Salt Stress Responses in Functional Soybean Nodules by High-Throughput Sequencing. Int J Mol Sci, 14(2), $2717-2738$.

Google Scholar: Author Only Title Only Author and Title

118. Sonia, C.B., Roseeta, D.M., Shivani, K., Santosh, K., Saloni, M., Saurabh, R. (2015) Insights into the Small RNA-Mediated Networks in Response to Abiotic Stress in Plants. Elucidation of Abiotic Stress Signaling in Plants, 45-91.

Google Scholar: Author Only Title Only Author and Title

119. Senthil, S., Yan F., Ramanjulu, S., W, B.B., Zhu, J.K., Oliver, Y. (2008) Novel and nodulation-regulated microRNAs in soybean roots. BMC genomics 9,160.

Google Scholar: Author Only Title Only Author and Title

120. Zhang, S.L., Wang, Y.N., Li, K.X., Zou, Y.M., Chen, L., Li, X. (2014) Identification of Cold-Responsive miRNAs and Their Target Genes in Nitrogen-Fixing Nodules of Soybean. INTERNATIONAL JOURNAL OF MOLECULAR SCIENCES, 15(8), 13596-13614.

Google Scholar: Author Only Title Only Author and Title

121. Kulcheski, F.R., de Oliveira, L.F., Molina, L.G. et al. (2011) Identification of novel soybean microRNAs involved in abiotic and biotic stresses. BMC Genomics, 12, 307.

Google Scholar: Author Only Title Only Author and Title

122. Supek, F., Bošnjak, M., Škunca, N., \& Šmuc, T. (2011). REVGO summarizes and visualizes long lists of gene ontology terms. PloS one, 6(7), e21800. 123. Lex, A, Gehlenborg, N., Strobelt, H., Vuillemot, R., \& Pfister, H. (2014). UpSet: Visualization of Intersecting Sets. IEEE transactions on visualization and computer graphics, 20(12), 1983-1992.

Google Scholar: Author Only Title Only Author and Title 